\title{
Crystallographic analysis of tryptophan halogenases AbeH and BorH
}

\author{
Md Ashaduzzaman, Kazi Lingkon, and John Bellizzi \\ Department of Chemistry and Biochemistry \\ College of Natural Sciences and Mathematics \\ The University of Toledo \\ 2801 W. Bancroft St., MS 602, Toledo OH 43606, USA \\ Md.Ashaduzzaman@rockets.utoledo.edu, Kazi.Lingkon@rockets.utoledo.edu
}

Flavin-dependent halogenases represent potential green catalysts for generating specific regioisomers of aryl halides for use in cross-coupling reactions and other synthetic applications [1]. They use $\mathrm{FADH}_{2}$ (provided by a separate flavin reductase component), $\mathrm{O}_{2}$, and $\mathrm{Cl}^{-}$to generate $\mathrm{HOCl}$, which transfers $\mathrm{Cl}^{+}$to an aromatic substrate in an electrophilic aromatic substitution reaction involving conserved Lys and Glu residues [2,3]. Structural analysis of several tryptophan halogenases suggests regioselectivity is dictated by the orientation of substrate in the active site, overriding any electronic effects [4].

$\mathrm{AbeH}$ and BorH were annotated as putative flavin-dependent tryptophan halogenases encoded by soil bacteria biosynthetic gene clusters producing the bisindole alkaloid natural products BE54017 [5] and borregomycin A [6] respectively. We have cloned, expressed, and purified $\mathrm{AbeH}$ and $\mathrm{BorH}$, and experimentally verified that both proteins have halogenase activity against L-Trp. BorH chlorinates L-Trp with $k_{\text {cat }}=4.4 \mathrm{~min}^{-1}$ and $K_{\mathrm{M}}=9.8 \mu \mathrm{M}$. NMR analysis of Cl-Trp produced by BorH confirmed regioselectivity for $\mathrm{C} 6$. BorH also can brominate L-Trp at C6.

Both $\mathrm{AbeH}$ and BorH have been crystallized. The AbeH-FAD complex crystallized in space group $\mathrm{P} 2{ }_{1} 2_{1} 2_{1}$, and the structure has been solved using molecular replacement and refined to $R_{\text {working }}=19 \%$ and $\mathrm{R}_{\text {free }}=21.7 \%$ at $1.65 \AA$ resolution (Fig. 1). BorH has crystallized in two different crystal forms (orthorhombic and hexagonal) and structure determination is in progress. Structural studies of both enzymes with bound L-Trp are ongoing with the goal of improving our understanding of the structural basis for regioselectivity in flavin-dependent halogenases.

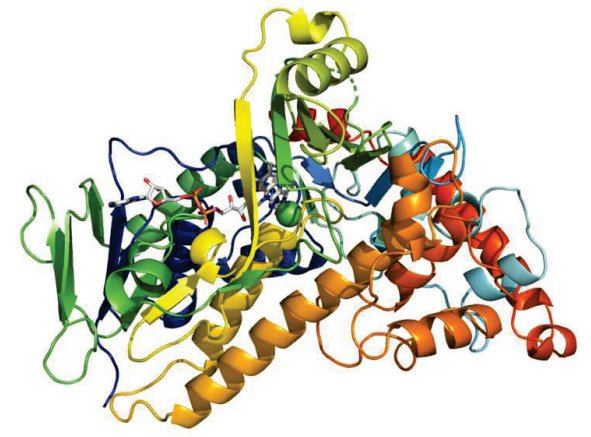

Fig. 1. AbeH:FAD complex

\section{References:}

1. Latham, J., et al.,(2018), Chemical Reviews 118, 232-269.

2. Dong, C., et al.,(2005), Science 309, 2216-2219.

3. $\quad$ Flecks, S., et al.,(2008), Angewandte Chemie 47, 9533-9536.

4. Ainsley, J., et al.,(2018), ACS Omega 3, 4847-4859.

5. $\quad$ Chang, F. Y.; Brady, S. F.,(2011), J Am Chem Soc 133, 9996-9999.

6. Chang, F. Y.; Brady, S. F.,(2013), Proc Natl Acad Sci U S A 110, 2478-2483. 